CONFORMAL GEOMETRY AND DYNAMICS

An Electronic Journal of the American Mathematical Society

Volume 14, Pages 167-183 (June 30, 2010)

S 1088-4173(2010)00209-2

\title{
CHECKING ATOMICITY OF CONFORMAL ENDING MEASURES FOR KLEINIAN GROUPS
}

\author{
KURT FALK, KATSUHIKO MATSUZAKI, AND BERND O. STRATMANN
}

\begin{abstract}
In this paper we address questions of continuity and atomicity of conformal ending measures for arbitrary non-elementary Kleinian groups. We give sufficient conditions under which such ending measures are purely atomic. Moreover, we will show that if a conformal ending measure has an atom which is contained in the big horospherical limit set, then this atom has to be a parabolic fixed point. Also, we give detailed discussions of nontrivial examples for purely atomic as well as for non-atomic conformal ending measures.
\end{abstract}

\section{INTRODUCTION AND STATEMENT OF THE RESULTS}

The idea of a conformal ending measure was originally sketched briefly by Sullivan in 21] for a Kleinian group with parabolic elements. Subsequently, this construction was investigated more rigorously and in greater generality in [1]. The construction of a conformal ending measure is very similar to the well-known Patterson measure construction (see [16]). However, in [8] it was shown that there are cases in which conformal ending measures cannot be derived via Patterson's construction. The basic idea of a conformal ending measure is as follows (see Section 3 for a more detailed review of conformal ending measures). Let $\left(z_{n}\right)$ be an ending sequence, that is, a sequence of points in the Poincaré ball model $(\mathbb{D}, d)$ of the hyperbolic space which converges radially to some point $\zeta \in \mathbb{S}=\partial \mathbb{D}$ within some Dirichlet fundamental domain of a Kleinian group $G$. If $\zeta$ is a limit point of $G$, then the closure of the orbit $G\left(z_{n}\right)$ converges to the limit set $L(G)$, for $n$ tending to infinity. Here, convergence is meant with respect to the Hausdorff metric on closed subsets of the closure $\overline{\mathbb{D}}=\mathbb{D} \cup \mathbb{S}$ in the Euclidean topology. If $s \in \mathbb{R}^{+}$is chosen such that the Poincaré series $\sum_{g \in G} j(g, z)^{s}$ of $G$ at $z \in \mathbb{D}$ with exponent $s$ converges, then there exist purely atomic $s$-conformal probability measures $\mu_{n}$ having their atoms in $G\left(z_{n}\right)$. Here, $j(g, z)$ denotes the conformal derivative of $g$ at $z \in \overline{\mathbb{D}}$, that is, the uniquely determined positive number such that $g^{\prime}(z) / j(g, z)$ is orthogonal, where $g^{\prime}(z)$ is the Jacobian of $g$ at $z$. For $\zeta \in \mathbb{S}$ we have $j(g, \zeta)=\left(1-\left|g^{-1}(0)\right|^{2}\right) /\left|\zeta-g^{-1}(0)\right|^{2}$ (see Section 4 for further details). Consequently, weak limits of these measures $\mu_{n}$ give rise to $s$-conformal measures supported on $L(G)$, and these measures are called s-conformal ending measures. The aim of this paper is to address questions of continuity and atomicity for this

Received by the editors March 18, 2009.

2010 Mathematics Subject Classification. Primary 30F40, 37F35; Secondary 37F30, 28A80.

The first author was supported by the Science Foundation Ireland.

The second author was supported by JSPS Grant B \#20340030.

(C)2010 American Mathematical Society Reverts to public domain 28 years from publication 
type of ending measures. Throughout we will use the phrase essential support of a measure to denote any measurable set of full measure (not necessarily closed nor uniquely determined), and $\mathbf{1}_{z}$ denotes the Dirac delta at $z$. The following statement summarises Theorem 4.3. Lemma 4.4, Proposition 4.5 and Theorem 4.6.

Let $\left(z_{n}\right)$ be a given ending sequence converging to $\zeta \in \mathbb{S}$, and assume that $j(h, \zeta)=1$ for every element $h$ of the stabiliser $\operatorname{Stab}_{G}(\zeta)$ of $\zeta$. Moreover, assume that the reduced horospherical Poincaré series $\sum_{g \in G / \operatorname{Stab}_{G}(\zeta)} j(g, \zeta)^{s}$ of $G$ at $\zeta$ with exponent s converges. We then have that the essential support of the associated s-conformal ending measure $\mu$ is equal to $G(\zeta)$. In particular, $\mu$ is purely atomic and coincides with the measure $\mu_{\zeta}$ given by

$$
\mu_{\zeta}=\frac{\sum_{g \in G / \operatorname{Stab}_{G}(\zeta)} j(g, \zeta)^{s} \mathbf{1}_{g(\zeta)}}{\sum_{g \in G / \operatorname{Stab}_{G}(\zeta)} j(g, \zeta)^{s}} .
$$

If $\zeta$ is an ordinary point of $G$ or a bounded parabolic fixed point of $G$, then the reduced horospherical Poincaré series of $G$ at $\zeta$ with exponent $s \in \mathbb{R}^{+}$ converges whenever the Poincaré series $\sum_{g \in G} j(g, z)^{s}, z \in \mathbb{D}$, converges.

This result provides us with typical examples of purely atomic conformal ending measures. Another class of examples is obtained by using a result of 11, stating that a conformal ending measure associated to a certain given end is essentially supported on the associated end limit set. We then have that if this end limit set is countable, then every ending measure essentially supported on it is necessarily purely atomic. Besides, this raises the question whether it is possible to have a countable end limit set associated to an end which does not originate from a parabolic fixed point. In fact, a result of Bishop [4, stating that a Kleinian group acting on three-dimensional hyperbolic space is geometrically finite if and only if the set of non-radial limit points is countable, strongly suggests that the case of parabolic cusps is the only case in which the end limit set of a given end is countable.

In Section 5 we first show that if a conformal ending measure for a Kleinian group $G$ has an atom which is contained in the big horospherical limit set $L_{H}(G)$ (see Section 2 for the definition), then this atom has to be a parabolic fixed point. Note that above we gave a sufficient condition, in terms of the convergence of the reduced horospherical Poincaré series at $\zeta$, under which a conformal ending measure is purely atomic. However, unless $\zeta$ is an ordinary point or $\zeta$ is a bounded parabolic fixed point, it is usually not an easy task to verify this convergence condition. Hence, it is desirable to find conditions which allow us to decide more easily whether a conformal ending measure has atoms or not. By employing a result of Tukia [22], which states that there always exists a measurable fundamental set for the action of a Kleinian group $G$ on the complement of $L_{H}(G)$, in Section 5 we show that if $G$ admits an $s$-conformal measure essentially supported on the dissipative part of the action of $G$ on $\mathbb{S}$, then we always have that there exists a purely atomic $s$-conformal ending measure for $G$. This observation then gives rise to the following result (see Theorem 5.4). Here, $\delta(G)$ denotes the abzissa of convergence of the Poincaré series of $G$.

For $s \geq \delta(G)$, we have that every s-conformal ending measure for $G$ supported on $L(G)$ is essentially supported on $L_{H}(G)$ if and only if every $s$ conformal ending measure for $G$ supported on $L(G)$ can have atoms only at parabolic fixed points of $G$. 
Eventually, in Section [6. we give various non-trivial examples of purely atomic and of non-atomic conformal ending measures. These include examples of infinitely generated Schottky groups whose conformal ending measures have atoms at Jørgensen points, and examples of non-atomic conformal ending measures on limit sets of normal subgroups of finitely generated Schottky groups. Moreover, we give the construction of a Kleinian group $\Gamma$ which admits a purely atomic $\delta(\Gamma)$-conformal ending measure at some Jørgensen point which is not a parabolic fixed point of $\Gamma$.

\section{Preliminaries}

Hyperbolic geometry in the $(N+1)$-dimensional ball model $(\mathbb{D}, d), N \geq 2$, and conformal geometry on the boundary sphere $\mathbb{S}$ have the same automorphism group, namely, the group $\operatorname{Con}(N)$ of conformal maps in $\mathbb{S}$ which is isomorphic to the group of orientation-preserving hyperbolic isometries of $\mathbb{D}$. (For $N=1$, Con(1) is merely defined by the latter group.) Any transformation in $\operatorname{Con}(N)$ gives rise to an isometry of $\mathbb{D}$, and vice versa. It is well known that this isomorphism arises naturally from the principle of Poincaré extension, based on the elementary observation that an $(N+1)$-dimensional hyperbolic half-space $H$ in $\mathbb{D}$ extends in a unique way to an $N$-dimensional ball $\bar{H} \cap \mathbb{S}$. A Kleinian group $G$ is a discrete subgroup of $\operatorname{Con}(N)$ and its limit set $L(G)$ is the derived set of an arbitrary $G$-orbit; that is, $L(G)=\overline{G(z)} \backslash G(z)$ for some arbitrary $z \in \mathbb{D}$. The limit set $L(G)$ is equal to the union of the set $L_{r}(G)$ of radial limit points (also called conical limit points) and the set $L_{t}(G)=L(G) \backslash L_{r}(G)$ of transient limit points (also called escaping limit points), where

$$
L_{r}(G)=\left\{\xi \in L(G): \liminf _{T \rightarrow \infty} \Delta\left(\xi_{T}\right)<\infty\right\} .
$$

Here, $\xi_{T}$ denotes the unique point on the hyperbolic geodesic ray from the origin $0 \in \mathbb{D}$ to $\xi$ for which $d\left(0, \xi_{T}\right)=T$, and $\Delta(z)=\inf \{d(z, g(0)): g \in G\}$ denotes the hyperbolic distance of $z \in \mathbb{D}$ to the orbit $G(0)$. Important subsets of $L(G)$ are the set $L_{u r}(G)$ of uniformly radial limit points and the set $L_{J}(G)$ of Jørgensen limit points. These are given as follows (cf. 7] and [15). $L_{u r}(G)$ is the set of $\xi \in L(G)$ for which $\limsup _{T \rightarrow \infty} \Delta\left(\xi_{T}\right)<\infty$, and $L_{J}(G)$ is the set of $\xi \in L(G)$ for which there exists a geodesic ray towards $\xi$ which is fully contained in some Dirichlet fundamental domain of $G$. One easily verifies that $L_{u r}(G) \subset L_{r}(G)$ and $L_{J}(G) \subset L_{t}(G)$. Next, recall that an element of $\operatorname{Con}(N)$ is called parabolic if it has precisely one fixed point in $\mathbb{S}$. If a Kleinian group $G$ contains parabolic elements, then the fixed points of these elements are contained in $L_{J}(G)$. A horosphere at $\zeta \in \mathbb{S}$ can be represented by the level set $h_{\zeta}(c)=\{z \in \mathbb{D}: k(z, \zeta)=c\}$ of the Poisson kernel $k(z, \zeta)=\left(1-|z|^{2}\right) /|\zeta-z|^{2}$, for some constant $c>0$. Clearly, $h_{\zeta}(c)$ is an Euclidean sphere tangent to $\mathbb{S}$ at $\zeta$ of radius equal to $1 /(1+c)$. The associated horoball $H_{\zeta}(c)$ is the open ball bounded by this horosphere; that is,

$$
H_{\zeta}(c)=\{z \in \mathbb{D}: k(z, \zeta)>c\} .
$$

Let $\operatorname{Stab}_{G}(\zeta)$ denote the stabiliser of $\zeta \in \overline{\mathbb{D}}$ in $G$ consisting of all elements of $G$ fixing $\zeta$. Clearly, if $\zeta$ is the fixed point of a parabolic element of $G$, then we have that any horoball at $\zeta$ is invariant under $\operatorname{Stab}_{G}(\zeta)$. Moreover, there is a constant $c>0$ such that $g\left(H_{\zeta}(c)\right) \cap H_{\zeta}(c)=\emptyset$, for every $g \in G \backslash \operatorname{Stab}_{G}(\zeta)$. A cusped region for $\zeta$ is given by the quotient $H_{\zeta}(c) / \operatorname{Stab}_{G}(\zeta)$ which we assume to be embedded in the hyperbolic orbifold $M_{G}=\mathbb{D} / G$. 
The convex hull $C(L(G))$ is the smallest closed convex subset of $\mathbb{D}$ whose Euclidean closure in $\overline{\mathbb{D}}$ contains $L(G)$. Equivalently, $C(L(G))$ is the hyperbolic convex hull of the union of all geodesic lines with endpoints in $L(G)$. Clearly, the set $C(L(G))$ is invariant under $G$, and hence, $C_{G}=C(L(G)) / G$ is a closed subset of $M_{G}=\mathbb{D} / G$. The set $C_{G}$ is called the convex core of $G$ or of $M_{G}$. A Kleinian group $G$ is called geometrically finite if there exists $\varepsilon>0$ such that the hyperbolic $\varepsilon$-neighbourhood of $C_{G}$ has finite hyperbolic volume. We say that a parabolic fixed point $\zeta$ of $G$ is bounded if the intersection of $C_{G}$ and $h_{\zeta}(c) / \operatorname{Stab}_{G}(\zeta)$ is compact. A theorem of Beardon and Maskit [3] asserts that $G$ is geometrically finite if and only if the limit set $L(G)$ is equal to the union of $L_{r}(G)$ and the set of bounded parabolic fixed points.

Let us also recall the following well-known basic formulae (see e.g. [14]). Let $j(g, z)$ denote the conformal derivative of $g \in \operatorname{Con}(N)$ at $z \in \overline{\mathbb{D}}$; that is,

$$
j(g, z)=\frac{1-\left|g^{-1}(0)\right|^{2}}{\left|z^{*}-g^{-1}(0)\right|^{2}|z|^{2}},
$$

where $z^{*}$ denotes the image of $z$ under the reflection at $\mathbb{S}$. An elementary calculation gives that if $\zeta \in \mathbb{S}$, then

$$
j(g, \zeta)=\frac{1-\left|g^{-1}(0)\right|^{2}}{\left|\zeta-g^{-1}(0)\right|^{2}},
$$

and hence, $j(g, \zeta)=k\left(g^{-1}(0), \zeta\right)$. Also, if $z \in \mathbb{D}$, then one finds

$$
j(g, z)=\frac{1-|g(z)|^{2}}{1-|z|^{2}} .
$$

For $z_{1}, z_{2} \in \mathbb{D}$, we define $d_{\zeta}\left(z_{1}, z_{2}\right)$ to be the signed hyperbolic distance between the horosphere at $\zeta$ through $z_{1}$ and the horosphere at $\zeta$ through $z_{2}$, where 'signed' refers to that $d_{\zeta}\left(z_{1}, z_{2}\right)$ is positive if $z_{2}$ is contained in the horoball bounded by the horosphere at $\zeta$ through $z_{1}$, and is negative otherwise. Clearly, $\left|d_{\zeta}\left(z_{1}, z_{2}\right)\right| \leq$ $d\left(z_{1}, z_{2}\right)$. It is well known that the relation between this horospherical distance and the Poisson kernel is given by

$$
d_{\zeta}(z, 0)=-\log k(z, \zeta)
$$

The Poincaré series associated with a Kleinian group $G$ is defined for $z \in \mathbb{D}$ and $s \in \mathbb{R}^{+}$by

$$
P(z, s)=\sum_{g \in G} j(g, z)^{s} .
$$

Note that convergence and divergence of $P(z, s)$ does not depend on the choice of $z \in \mathbb{D}$. The exponent of convergence of $G$ is then given by

$$
\delta(G)=\inf \left\{s \in \mathbb{R}^{+}: P(z, s)<\infty\right\} .
$$

If $P(z, s)$ converges at $s=\delta(G)$; that is, if $P(z, \delta(G))<\infty$, then $G$ is said to be of convergence type. Otherwise, $G$ is said to be of divergence type. Note that in 5 . it was shown that if $G$ is some arbitrary non-elementary Kleinian group, then the Hausdorff dimension of $L_{r}(G)$ and of $L_{u r}(G)$ are both equal to $\delta(G)$ (see also [17]).

Also, recall that a Borel probability measure $\mu$ on $\overline{\mathbb{D}}$ is called $s$-conformal for a Kleinian group $G$ if, for each $g \in G$ and any measurable set $A$ on $\overline{\mathbb{D}}$,

$$
\mu(g(A))=\int_{A} j(g, z)^{s} d \mu(z) .
$$


This condition is equivalent to that for each continuous function $f$ on $\overline{\mathbb{D}}$ and for each $g \in G$, we have

$$
\int_{\overline{\mathbb{D}}} f\left(g^{-1}(z)\right) d \mu(z)=\int_{\overline{\mathbb{D}}} f(z) j(g, z)^{s} d \mu(z) .
$$

For a Kleinian group $G$, the big horospherical limit set $L_{H}(G)$ consists of all points $\zeta \in L(G)$ with the property that there is a constant $c>0$ such that the orbit $G(0)$ accumulates at $\zeta$ within the horoball $H_{\zeta}(c)$. It is easy to see that $L_{r}(G) \subset L_{H}(G)$ and that parabolic fixed points of $G$ are contained in $L_{H}(G)$.

It is known that a Kleinian group $G$ acts conservatively on $L_{H}(G)$ with respect to any conformal measure $\mu$ for $G$ (see Tukia [22]). This means that there are infinitely many elements $g \in G$ such that $\mu(A \cap g(A))>0$, for each measurable set $A \subset L_{H}(G)$ for which $\mu(A)>0$. On the other hand, Tukia [22] also showed that $G$ acts dissipatively on the complement $\mathbb{S} \backslash L_{H}(G)$; that is, there exists a measurable fundamental set $E \subset \mathbb{S} \backslash L_{H}(G)$ such that $G(\zeta) \cap E$ is a singleton, for each $\zeta \in \mathbb{S} \backslash L_{H}(G)$. These results provide significant generalisations of a theorem of Sullivan [20, who derived these assertions for the special case in which $\mu$ is equal to the $N$-dimensional Lebesgue measure $m$. Next, recall that the big Dirichlet set $D(G)$ of $G$ is equal to the set of all points that lie in the boundary at infinity of some Dirichlet fundamental domain. Clearly, $D(G)$ can be written as a disjoint union as follows:

$$
D(G)=\Omega(G) \cup L_{J}(G) .
$$

It is known that $D(G) \cup L_{H}(G)=\mathbb{S}$ and that $m\left(D(G) \cap L_{H}(G)\right)=0$ (see [11]).

Finally, let us also recall from [1] that a connected open subset $E$ of $M_{G}=\mathbb{D} / G$ is called an end of $M_{G}$, if $\partial E$ is compact and non-empty and if $E$ has non-compact closure in $M_{G}$. For a suitable index set $I$, let $\left\{E_{i}^{0}: i \in I\right\}$ denote the set of all connected components of the lift of $E$ to $\mathbb{D}$, and let $G_{i}$ denote the stabiliser $\operatorname{Stab}_{G}\left(E_{i}^{0}\right)$ of $E_{i}^{0}$ in $G$. The set $E_{i}^{0}$ is called an end of $G$, and $G_{i}$ the corresponding end group of $G$. By construction, the $G_{i}$ are subgroups of $G$ which are all conjugate to each other. A point $\zeta \in \mathbb{S}$ is called an endpoint of an end $E_{i}^{0}$ of $G$ if, whenever $\beta$ is a hyperbolic ray towards $\zeta$, there exists a subray $\beta^{\prime}$ of $\beta$ that is contained in $E_{i}^{0}$, for which the hyperbolic distance $d\left(z, \partial E_{i}^{0}\right)$ tends to infinity as $z$ approaches $\zeta$ on $\beta^{\prime}$. Note that the set of endpoints of $E_{i}^{0}$ has empty intersection with $L_{r}(G)$. If additionally $\zeta \in L\left(G_{i}\right)$, then $\zeta$ is called the end limit point of $E_{i}^{0}$. Note that the set of end limit points of $E_{i}^{0}$ is contained in $L\left(G_{i}\right) \backslash L_{r}(G)$. The union of all end limit points of all ends $E_{i}^{0}, i \in I$, shall be referred to as the end limit set of $G$ associated with $E$, or simply as the end limit set of $E$.

So far, ends have been subsets $E$ of $M_{G}$ (or of the lift to $\mathbb{D}$ of such a set) such that $E$ is a non-compact component of $M_{G} \backslash \partial E$, where the boundary $\partial E$ of $E$ in $M_{G}$ is compact. Note that the definition of an end can also be given in terms of $\bar{M}_{G}=(\mathbb{D} \cup \Omega(G)) / G$. Namely, in these terms, $E$ is a non-compact component of $\bar{M}_{G} \backslash \bar{\partial} E$, where the boundary $\bar{\partial} E$ taken in $\bar{M}_{G}$ is compact. In 1 such a set $E$ was called an 'end with boundary'. Note that for ends with boundary one has by [1. Theorem 4.4] that there exists a non-trivial conformal measure supported by the end limit point set of an end with boundary. Moreover, [1, Proposition 4.5] then allows us to extend this conformal measure for an end group to a conformal measure for $G$. We shall make use of this observation in the proof of Proposition 6.2 of this paper. For further details on end limit sets and ends with boundary we refer to [1] and [8]. 


\section{The COnstruction of CONFORMAL ENDing MeAsures}

In order to recall the construction of an $s$-conformal ending measure, let $F$ be a Dirichlet fundamental domain of some given Kleinian group $G$, and let $\bar{F}$ be its closure in $\overline{\mathbb{D}}$. A sequence $\left(z_{n}\right)$ of elements $z_{n} \in F$ is called an ending sequence tending to $\zeta \in \bar{F} \cap \mathbb{S}$, if each of the $z_{n}$ lies within a bounded distance from some geodesic ray towards $\zeta$ contained in $F$, and if the sequence $\left(z_{n}\right)$ tends to $\zeta$ with respect to the Euclidean metric. Let $s \in \mathbb{R}^{+}$be chosen such that $P(z, s)<\infty$. For a given ending sequence $\left(z_{n}\right)$ tending to $\zeta \in \bar{F} \cap \mathbb{S}$, we define, for each $n \in \mathbb{N}$, the point mass $\mu_{n}$ on the closed unit ball $\overline{\mathbb{D}}$ as follows:

$$
\mu_{n}=\frac{1}{P\left(z_{n}, s\right)} \sum_{g \in G} j\left(g, z_{n}\right)^{s} \mathbf{1}_{g\left(z_{n}\right)} .
$$

The Helly-Bray Theorem then ensures that, by passing to a subsequence if necessary, the sequence $\left(\mu_{n}\right)$ has a weak limit measure $\mu$, and we will refer to this measure as the $s$-conformal ending measure. By construction, for each of the $\mu_{n}$ we have, for any $g \in G$ and for any continuous function $f$ on $\overline{\mathbb{D}}$,

$$
\begin{aligned}
\int_{\overline{\mathbb{D}}} f\left(g^{-1}(z)\right) d \mu_{n}(z) & =\frac{1}{P\left(z_{n}, s\right)} \sum_{h \in G} f\left(g^{-1} h\left(z_{n}\right)\right) j\left(h, z_{n}\right)^{s} \\
& =\frac{1}{P\left(z_{n}, s\right)} \sum_{h \in G} f\left(h\left(z_{n}\right)\right) j\left(g h, z_{n}\right)^{s} \\
& =\frac{1}{P\left(z_{n}, s\right)} \sum_{h \in G} f\left(h\left(z_{n}\right)\right) j\left(g, h\left(z_{n}\right)\right)^{s} j\left(h, z_{n}\right)^{s} \\
& =\int_{\overline{\mathbb{D}}} f(z) j(g, z)^{s} d \mu_{n}(z) .
\end{aligned}
$$

Since $\left(\mu_{n}\right)$ converges weakly to $\mu$, it therefore follows that $\mu$ satisfies the same $s$-conformal transformation rule. Therefore, we have, for all $g \in G$ and $A \subset \overline{\mathbb{D}}$ measurable,

$$
\mu(g(A))=\int_{A} j(g, z)^{s} d \mu .
$$

Finally, let us remark that if $L_{J}(G)$ is non-empty and if $\mu$ is an $s$-conformal ending measure derived from an ending sequence $\left(z_{n}\right)$ tending towards some $\zeta \in$ $L_{J}(G)$, then the support of $\mu$ has to be equal to $L(G)$. Indeed, consider any open set $U \subset \overline{\mathbb{D}}$ whose closure $\bar{U}$ is contained in $\overline{\mathbb{D}} \backslash L(G)$. The discontinuous action of $G$ on $\overline{\mathbb{D}} \backslash L(G)$ immediately implies that there exist at most finitely many $g \in G$ such that $g(F) \cap U \neq \emptyset$. Furthermore, we have that $g(\bar{F}) \cap \bar{U}$ is compact in $\overline{\mathbb{D}} \backslash L(G)$, for each $g \in G$. Since the sequence $\left(z_{n}\right)$ used in the definition of $\mu_{n}$ was chosen to be an ending sequence contained in $F$, it then follows that there exists $n_{U} \in \mathbb{N}$ such that $\mu_{n}(U)=0$, for all $n \geq n_{U}$. This shows that $\mu(U)=0$, and hence, $\mu$ is supported on $L(G)$.

\section{Convergence AT Infinity And Purely ATOMiC Measures}

Throughout, let $G$ be a Kleinian group. As already mentioned briefly in the introduction, extensions of the Poincaré series $P(z, s)$ to points in $\mathbb{S}$ gives rise to what we call the horospherical Poincaré series of $G$. More precisely, the horospherical 
Poincaré series $\mathcal{P}(\zeta, s)$ of $G$ at $\zeta \in \mathbb{S}$ with exponent $s \in \mathbb{R}^{+}$is given by

$$
\mathcal{P}(\zeta, s)=\sum_{g \in G} j(g, \zeta)^{s}
$$

Note that Morosawa [13] also considered this type of horospherical Poincaré series and obtained basic results concerning atoms of conformal measures, some of which will be generalised in this paper. Also, we refer to [14] (Section 3.5) for some basic facts concerning the atomic part of a conformal measure.

Let us first prove the following two elementary lemmata. These will turn out to be useful later.

Lemma 4.1. If $\mathcal{P}(\zeta, s)<\infty$ for some $\zeta \in \mathbb{S}$, then $P(z, s)<\infty$ for every $z \in \mathbb{D}$. In particular, we hence have that if $\mathcal{P}(\zeta, s)<\infty$ for some $\zeta \in \mathbb{S}$, then $s \geq \delta(G)$. Moreover, if $P(z, s)<\infty$ for some (and hence for all) $z \in \mathbb{D}$, then $\mathcal{P}(\zeta, s)<\infty$ for each $\zeta \in \Omega(G)$.

Proof. Using the identities for the conformal derivative $j(g, \cdot)$ stated in Section 2, one immediately verifies that for $\zeta \in \mathbb{S}$ and $z \in \mathbb{D}$ we have

$$
j(g, \zeta)=\frac{\left|z^{*}-g^{-1}(0)\right|^{2}|z|^{2}}{\left|\zeta-g^{-1}(0)\right|^{2}} j(g, z) \geq \frac{(1-|z|)^{2}}{4} j(g, z) .
$$

This gives the first statement in the lemma. For the second statement note that if $\zeta \in \Omega(G)$, then $\left|\zeta-g^{-1}(0)\right|^{2} \geq c$, for some constant $c>0$ not depending on $g \in G$. Hence,

$$
j(g, \zeta) \leq \frac{(1+|z|)^{2}}{c} j(g, z),
$$

which gives the second statement in the lemma.

Lemma 4.2. Let $\left(z_{n}\right)$ be a sequence in $\mathbb{D}$ which converges to $\zeta \in \mathbb{S}$. Then the following hold:

(i) We always have $\liminf _{n \rightarrow \infty} P\left(z_{n}, s\right) \geq \mathcal{P}(\zeta, s)$, and therefore, if $\mathcal{P}(\zeta, s)$ diverges, then $\lim _{n \rightarrow \infty} P\left(z_{n}, s\right)=\infty$.

(ii) If $\zeta \in \Omega(G)$, then $\lim _{n \rightarrow \infty} P\left(z_{n}, s\right)=\mathcal{P}(\zeta, s)$.

(iii) If $\left(z_{n}\right)$ converges radially to $\zeta \in \mathbb{S}$, then $\lim _{n \rightarrow \infty} P\left(z_{n}, s\right)=\mathcal{P}(\zeta, s)$.

Proof. The statement in (i) is an immediate consequence of Fatou's Lemma. For the remaining statements, we can assume without loss of generality that $\mathcal{P}(\zeta, s)$ converges. If $\zeta \in \Omega(G)$, then clearly $\left|\zeta-g^{-1}(0)\right|^{2}$ is uniformly bounded away from 0 , for all $g \in G$. Therefore, since $\left|z_{n}^{*}-g^{-1}(0)\right|^{2}$ tends to $\left|\zeta-g^{-1}(0)\right|^{2}$ for $n$ tending to infinity, there exists a constant $c_{1}>0$ such that, for each $g \in G$ and every $n \in \mathbb{N}$,

$$
\left|z_{n}^{*}-g^{-1}(0)\right|^{2}\left|z_{n}\right|^{2} \geq c_{1}\left|\zeta-g^{-1}(0)\right|^{2} .
$$

Hence, $j\left(g, z_{n}\right)^{s} \leq c_{1}^{-s} j(g, \zeta)^{s}$. For the remaining assertion, assume that $z_{n}$ converges radially to $\zeta$, in the sense that each $z_{n}$ is at most a uniformly bounded distance away from some geodesic ray ending at $\zeta$. This immediately implies that the angle $\theta$ at $\zeta$ in the Euclidean triangle with vertices $g^{-1}(0), \zeta$ and $z_{n}^{*}$ is uniformly bounded away from zero. An application of the Euclidean sine rule then gives us that $\left|\zeta-g^{-1}(0)\right| \leq(1 / \sin \theta)\left|z_{n}^{*}-g^{-1}(0)\right|$, for all $n \in \mathbb{N}$ and $g \in G$. Thus, there exists a constant $c_{2}>0$ such that, for each $g \in G$ and every $n \in \mathbb{N}$,

$$
\left|z_{n}^{*}-g^{-1}(0)\right|^{2}\left|z_{n}\right|^{2} \geq c_{2}\left|\zeta-g^{-1}(0)\right|^{2} .
$$


This implies that $j\left(g, z_{n}\right)^{s} \leq c_{2}^{-s} j(g, \zeta)^{s}$. Since we have by definition that $j\left(g, z_{n}\right)$ tends to $j(g, \zeta)$ for $n$ tending to infinity, the dominated convergence theorem now gives in the situation of (ii) and of (iii) that $P\left(z_{n}, s\right)$ tends to $\mathcal{P}(\zeta, s)$ for $n$ tending to infinity.

The following theorem shows that convergence and divergence of the horospherical Poincaré series is crucial for finding out whether $s$-conformal ending measures have atoms or not.

Theorem 4.3. Let $\left(z_{n}\right)$ be a given ending sequence which tends to $\zeta$, for some $\zeta \in D(G)=\Omega(G) \cup L_{J}(G)$ not fixed by any non-trivial element of $G$. Also, suppose that $\mathcal{P}(\zeta, s)$ converges for $s \geq \delta(G)$. We then have that the essential support of the associated s-conformal ending measure $\mu$ is equal to $G(\zeta)$. In particular, $\mu$ is purely atomic and coincides with

$$
\mu_{\zeta}=\frac{1}{\mathcal{P}(\zeta, s)} \sum_{g \in G} j(g, \zeta)^{s} \mathbf{1}_{g(\zeta)}
$$

Proof. Let $K=\left\{z_{1}, z_{2}, \ldots\right\} \cup\{\zeta\}$ and consider

$$
\mu_{n}=\frac{1}{P\left(z_{n}, s\right)} \sum_{g \in G} j\left(g, z_{n}\right)^{s} \mathbf{1}_{g\left(z_{n}\right)} .
$$

Clearly $K$ is a compact subset of $\overline{\mathbb{D}}$. Combining this with the observation that $\limsup _{n \rightarrow \infty} \mu_{n}(C) \leq \mu(C)$ for each $C \subset \overline{\mathbb{D}}$ compact, which is an immediate consequence of weak convergence, we obtain

$$
\begin{aligned}
1 & \geq \mu(G(\zeta))=\sum_{h \in G} \mu(h(K)) \geq \sum_{h \in G} \limsup _{n \rightarrow \infty} \mu_{n}(h(K)) \\
& =\sum_{h \in G} \limsup _{n \rightarrow \infty} \frac{j\left(h, z_{n}\right)^{s}}{P\left(z_{n}, s\right)} .
\end{aligned}
$$

By Lemma 4.2, we have

$$
\lim _{n \rightarrow \infty} P\left(z_{n}, s\right)=\mathcal{P}(\zeta, s) .
$$

Since $\mathcal{P}(\zeta, s)$ converges by assumption, the last term in the above chain of inequalities is equal to 1 . This shows that $\mu(G(\zeta))=1$, and thus the probability measure $\mu$ is essentially supported on the countable set $G(\zeta)$.

Note that the statement of Theorem 4.3 includes the case in which $\zeta \in \Omega(G)$. In this case the resulting measure $\mu_{\zeta}=\frac{1}{\mathcal{P}(\zeta, s)} \sum_{g \in G} j(g, \zeta)^{s} \mathbf{1}_{g(\zeta)}$ is a conformal measure in the sense we use here (see Section 2), although strictly speaking it is not supported on the limit set of $G$.

Next we consider the case in which $\zeta$ is a parabolic fixed point of $G$. In this situation we have that the horospherical Poincaré series $\mathcal{P}(\zeta, s)$ at $\zeta$ diverges, for every $s \in \mathbb{R}$. This follows, since $j(g, \zeta)=e^{-d_{\zeta}\left(g^{-1}(0), 0\right)}$ is constant for infinitely many $g \in G$. In this situation we then consider the reduced horospherical Poincaré series

$$
\mathcal{P}_{\text {red }}(\zeta, s)=\sum_{g \in G / \operatorname{Stab}_{G}(\zeta)} j(g, \zeta)^{s}
$$


and if this series converges, then we have that the associated $s$-conformal measure

$$
\mu_{\zeta}=\frac{1}{\mathcal{P}_{\text {red }}(\zeta, s)} \sum_{g \in G / \operatorname{Stab}_{G}(\zeta)} j(g, \zeta)^{s} \mathbf{1}_{g(\zeta)}
$$

is purely atomic. Note that $\mu_{\zeta}$ is indeed an $s$-conformal measure for $G$, as a computation similar to the one in Section 3 readily shows. For the case in which $\zeta$ is a bounded parabolic fixed point, the following lemma shows that if the Poincaré series converges, then the reduced horospherical Poincaré series converges as well. Therefore, if $s>\delta(G)$ or if $s=\delta(G)$ for $G$ of convergence type, then we always have that there exists a purely atomic $s$-conformal measure essentially supported on $G(\zeta)$.

Lemma 4.4. For $\zeta$ a bounded parabolic fixed point of a Kleinian group $G$, we have that if $P(z, s)$ converges for $s \geq \delta(G)$, then so does $\mathcal{P}_{\text {red }}(\zeta, s)$.

Proof. Let $c>0$ be chosen such that $H_{\zeta}(c) / \operatorname{Stab}_{G}(\zeta)$ is a cusped region in $M_{G}$. Without loss of generality, we may assume $0 \in h_{\zeta}(c) \cap C(L(G))$. Since $\zeta$ is a bounded parabolic fixed point, there exists a constant $b>0$ such that $d\left(0,\left(\operatorname{Stab}_{G}(\zeta)\right)(z)\right) \leq$ $b$, for each $z \in h_{\zeta}(c) \cap C(L(G))$. Then choose representatives $\gamma \in G$ of the cosets in $G / \operatorname{Stab}_{G}(\zeta)$ such that

$$
d(0, \gamma(0))=\min \left\{d(0, g(0)): g \in \gamma \operatorname{Stab}_{G}(\zeta)\right\}
$$

With this choice, we then have, for each of these coset representatives $\gamma$,

$$
e^{-d_{\zeta}\left(\gamma^{-1}(0), 0\right)} \leq e^{b} e^{-d\left(\gamma^{-1}(0), 0\right)}
$$

Since $j(g, \zeta)=e^{-d_{\zeta}\left(\gamma^{-1}(0), 0\right)}$ for every $g \in \gamma \operatorname{Stab}_{G}(\zeta)$, it follows that

$$
\mathcal{P}_{\text {red }}(\zeta, s)=\sum_{\gamma \in G / \operatorname{Stab}_{G}(\zeta)} e^{-s d_{\zeta}\left(\gamma^{-1}(0), 0\right)} \leq e^{s b} \sum_{\gamma \in G / \operatorname{Stab}_{G}(\zeta)} e^{-s d\left(\gamma^{-1}(0), 0\right)} \leq e^{s b} P(0, s) .
$$

This finishes the proof of the lemma.

We now look at the more general situation in which there exists a purely atomic $s$-conformal measure essentially supported on $G(\zeta)$ for some point $\zeta \in \mathbb{S}$. Note that the measures considered in the following proposition are conformal measures which are not necessarily ending measures as introduced in Section 3 .

Proposition 4.5. Let $G$ be a Kleinian group, and let $\zeta \in \mathbb{S}$ and $s \in \mathbb{R}^{+}$be fixed. Then there exists a purely atomic s-conformal measure $\mu_{\zeta}$ essentially supported on $G(\zeta)$ if and only if

$$
j(h, \zeta)=1 \text { for each } h \in \operatorname{Stab}_{G}(\zeta) \text {, and } \mathcal{P}_{\text {red }}(\zeta, s)<\infty .
$$

Proof. Without loss of generality we may assume that $G$ has no elliptic elements. This follows mainly since $j(h, \zeta)=1$ for any fixed point $\zeta$ of an elliptic element $h \in G$, and since $\operatorname{Stab}_{G}(\zeta)$ has a subgroup of finite index with no elliptic elements. The details are left to the reader.

If $j(h, \zeta)=1$, for each $h \in \operatorname{Stab}_{G}(\zeta)$, and if the reduced horospherical Poincaré series converges, then $\mu_{\zeta}$ is a purely atomic conformal measure. Here, $\mu_{\zeta}$ is defined as prior to Lemma 4.4. This gives one direction of the equivalence. For the reverse direction, assume that there exists a purely atomic conformal measure $\mu$ essentially supported on $G(\zeta)$. Let us split the argument into the two cases: $\operatorname{Stab}_{G}(\zeta)$ is trivial and $\operatorname{Stab}_{G}(\zeta)$ is non-trivial. If $\operatorname{Stab}_{G}(\zeta)$ is trivial, then the $s$-conformality 
of $\mu$ implies that $\mu(\{g \zeta\})=j(g, \zeta)^{s} \mu(\{\zeta\})$. Thus, $\mu=\sum_{g \in G} j(g, \zeta)^{s} \mu(\{\zeta\}) \mathbf{1}_{g(\zeta)}$, and therefore, since $\mu$ is a probability measure,

$$
\sum_{g \in G} j(g, \zeta)^{s}=1 / \mu(\{\zeta\})<\infty .
$$

If $\operatorname{Stab}_{G}(\zeta)$ is non-trivial, then $\zeta$ is either a parabolic or a loxodromic fixed point. If $\zeta$ is a loxodromic fixed point of $G$, then a conformal measure cannot have an atom at $\zeta$. This follows, since $j(h, \zeta) \neq 1$ for each loxodromic element $h \in G$ fixing $\zeta$. Therefore, $\zeta$ has to be a parabolic fixed point. In this situation we have that $j(h, \zeta)=1$, for each $h \in \operatorname{Stab}_{G}(\zeta)$. Arguing in a similar way as in the trivial stabiliser case, it follows that the reduced horospherical Poincaré series converges.

Combining Theorem 4.3 and Proposition 4.5, the statement of Theorem 4.3 can be extended to the case in which $\operatorname{Stab}_{G}(\zeta)$ is non-trivial. The proof is straightforward and left to the reader.

Theorem 4.6. For a Kleinian group $G$, let $\left(z_{n}\right)$ be an ending sequence converging to $\zeta \in D(G)$, and let $\mu$ be the associated s-conformal ending measure. Then $\mu$ has an atom at $\zeta$ if and only if

$$
j(h, \zeta)=1 \text { for each } h \in \operatorname{Stab}_{G}(\zeta) \text {, and } \mathcal{P}_{\text {red }}(\zeta, s)<\infty .
$$

In case the latter is satisfied, we then in particular have that $\mu$ coincides with the purely atomic s-conformal measure

$$
\mu_{\zeta}=\frac{1}{\mathcal{P}_{\text {red }}(\zeta, s)} \sum_{g \in G / \operatorname{Stab}_{G}(\zeta)} j(g, \zeta)^{s} \mathbf{1}_{g(\zeta)},
$$

which is essentially supported on $G(\zeta)$.

Let us also briefly mention another way to produce purely atomic conformal ending measures. For this, let us assume that the end limit set associated to some end of a hyperbolic manifold $M_{G}=\mathbb{D} / G$ is countable, for some Kleinian group $G$. Here, the notions of end and end limit set are adopted from [1] (see also Section 2). Theorem 4.4 in [1] then ensures that the type of ending measure constructed in the previous section is essentially supported on the corresponding end limit set, and hence, this measure is necessarily purely atomic. Recall the basic definitions concerning ends of hyperbolic manifolds given in Section 2, in particular the notion of end limit set of $G$ associated with some end $E$ of $M_{G}$.

Proposition 4.7. Let $G$ be a Kleinian group such that $M_{G}$ has an end E. Furthermore, assume that the end limit set associated with $E$ is countable. Then every conformal ending measure obtained from any ending sequence converging to any end limit point of any end $E$ is purely atomic.

Bishop [4] improved a classical result of Beardon and Maskit [3], by showing that a Kleinian group $G$ acting on hyperbolic 3-space is geometrically finite if and only if $L(G) \backslash L_{r}(G)$ is countable (possibly empty). It is not difficult to see that this result continues to hold for Fuchsian groups acting on hyperbolic 2-space. Then, a combination of this result of Bishop and Proposition 4.7 strongly supports the following conjecture. (Note that this conjecture is certainly true in two and three dimensions.) 
Conjecture. The end limit set of an end is countable if and only if it consists of finitely many orbits of parabolic fixed points.

Remark 4.8. Note that if the latter conjecture holds, then this would imply that the class of examples mentioned prior to Lemma 4.4 represents the only class of purely atomic conformal ending measures emerging from Proposition 4.7.

\section{Conformal ending measures AND the Big horospherical Limit Set}

In this section we show that if $\zeta$ is not fixed by any parabolic element of a Kleinian group $G$ and if $\zeta$ is an atom of some conformal ending measure for $G$, then $\zeta$ lies in the dissipative part of the action of $G$ on $\mathbb{S}$. Moreover, we will see that if the dissipative part of $L(G)$ has positive mass for some conformal measure, then there exists an atomic conformal ending measure for $G$.

Recall that a Kleinian group $G$ is said to act dissipatively, on some measurable $G$-invariant set $A$, if there exists a measurable set $E \subset A$ such that $E \cap G(\zeta)$ is a singleton, for each $\zeta \in A$. Note that the dissipative action is defined independently of conformal measures. Let $L_{H}(G)$ be the big horospherical limit set of $G$ and consider the complement $\mathbb{S} \backslash L_{H}(G)$, which is clearly $G$-invariant and contained in the big Dirichlet set $D(G)=\Omega(G) \cup L_{J}(G)$. In [22, Tukia showed that $G$ acts dissipatively on $\mathbb{S} \backslash L_{H}(G)$. Moreover, in 22 it was also shown that if $G$ acts dissipatively on a $G$-invariant set $A$, then $A$ is contained in $\mathbb{S} \backslash L_{H}(G)$ up to parabolic fixed points of $G$ and up to sets of measure zero, for any conformal measure for $G$. In this sense, we can say that $\mathbb{S} \backslash L_{H}(G)$ is the maximal subset of $\mathbb{S}$ on which $G$ acts dissipatively, and therefore we can say that $\mathbb{S} \backslash L_{H}(G)$ represents the dissipative part of the action of $G$ on $\mathbb{S}$.

Proposition 5.1. For a Kleinian group $G$ we have that if $\zeta \in L_{H}(G)$ is not a parabolic fixed point of $G$, then $\zeta$ is not an atom of $\mu$, for any s-conformal measure $\mu$ for $G$.

Proof. If $\zeta \in L_{H}(G)$, then there is a sequence $\left(g_{n}^{-1}\right)$ of elements $g_{n}^{-1} \in G$ such that $\left(g_{n}^{-1}(0)\right)$ converges to $\zeta$ within a horoball $H_{\zeta}(c)$ for some $c>0$. In this case, we have $j\left(g_{n}, \zeta\right)>c$, for all $n \in \mathbb{N}$. Combining this with Proposition 4.5, it follows that $\zeta$ can be an atom only if $\operatorname{Stab}_{G}(\zeta)$ is infinite and if $j(h, \zeta)=1$, for every $h \in \operatorname{Stab}_{G}(\zeta)$. However, this is only possible if $\zeta$ is a parabolic fixed point of $G$.

Remark 5.2. Note that the intersection $L_{J}(G) \cap L_{H}(G)$ can contain a point $\zeta$ which is not a parabolic fixed point. In this case, the horospherical Poincaré series $\mathcal{P}(\zeta, s)$ at $\zeta$ diverges for every exponent $s \in \mathbb{R}^{+}$. Indeed, if it would converge, then Theorem 4.3 guarantees the existence of a purely atomic $s$-conformal ending measure $\mu$ with an atom at $\zeta$, contradicting Proposition 5.1. An example of such a point $\zeta \in L_{J}(G) \cap L_{H}(G)$ can be given by taking a normal subgroup $G$ of some Kleinian group $\Gamma$. By choosing a suitable hyperbolic element $\gamma \in \Gamma$ with $G \cap\langle\gamma\rangle=$ $\{$ id.\}, we can guarantee on the one hand that the fixed point $\zeta$ of $\gamma$ is contained in the boundary at infinity of some Dirichlet fundamental domain of $G$. On the other hand, by a result of [10] we have that $\zeta \in L_{r}(\Gamma) \subset L_{H}(G)$.

The following proposition shows that if there exists a conformal measure which gives positive mass to the dissipative part, then there also exists a purely atomic conformal ending measure. This result relies on Tukia's results mentioned above. 
Proposition 5.3. If there exists an s-conformal measure $\mu$ for $G$ such that $\mu\left(A \backslash L_{H}(G)\right)>0$, for some $G$-invariant measurable set $A$, then there exists a purely atomic s-conformal ending measure $\nu$ for $G$ essentially supported on $A$ such that each atom of $\nu$ is not fixed by any parabolic element of $G$.

Proof. Let $A$ be $G$-invariant and measurable such that $\mu\left(A \backslash L_{H}(G)\right)>0$. By [22], we then have that there exists a measurable set $E \subset A \backslash L_{H}(G)$ such that $G(\zeta)$ has exactly one point in $E$, for each $\zeta \in A \backslash L_{H}(G)$. Using $\mu\left(A \backslash L_{H}(G)\right)>0$, it follows that $\mu(E)>0$. Define a measurable subset $E_{n}$, for each positive integer $n$, by

$$
E_{n}=\left\{\zeta \in E: \# \operatorname{Stab}_{G}(\zeta) \leq n\right\} .
$$

Remark that non-elliptic fixed points belong to $L_{H}(G)$. Since $E=\bigcup_{n=1}^{\infty} E_{n}$ and $\mu(E)>0$, there exists some $n$ such that $\mu\left(E_{n}\right)>0$. Hence,

$$
1 \geq \mu\left(A \backslash L_{H}(G)\right) \geq \mu\left(\bigcup_{g \in G} g\left(E_{n}\right)\right) \geq \frac{1}{n} \sum_{g \in G} \mu\left(g\left(E_{n}\right)\right) .
$$

Since $\mu$ is an $s$-conformal measure for $G$, the final term in the latter chain of inequalities is equal to

$$
\frac{1}{n} \sum_{g \in G} \int_{E_{n}} j(g, \zeta)^{s} d \mu(\zeta)=\frac{1}{n} \int_{E_{n}} \mathcal{P}(\zeta, s) d \mu(\zeta) .
$$

This implies that $\mathcal{P}(\zeta, s)$ converges, for $\mu$-almost every $\zeta \in E_{n}$. In particular, there exists some $\xi \in E_{n}$ such that $\mathcal{P}_{\text {red }}(\xi, s)<\infty$. Since $\xi \in D(G)$, Theorem 4.6 shows that the measure

$$
\nu=\frac{1}{\mathcal{P}_{\text {red }}(\xi, s)} \sum_{g \in G / \operatorname{Stab}_{G}(\xi)} j(g, \xi)^{s} \mathbf{1}_{g(\xi)}
$$

is a purely atomic $s$-conformal ending measure. In particular, since $\operatorname{Stab}_{G}(\xi)$ is finite, we have that $\xi$ is not fixed by any parabolic element of $G$.

We now summarize the two previous propositions in the following theorem.

Theorem 5.4. For a Kleinian group $G$ and for $s \geq \delta(G)$, the following are equivalent.

(i) Every s-conformal ending measure for $G$ supported on $L(G)$ is essentially supported on $L_{H}(G)$.

(ii) Every s-conformal ending measure for $G$ supported on $L(G)$ can have atoms only at parabolic fixed points of $G$.

Proof. Let $\zeta \in L(G)$ be given such that $\zeta$ is not a parabolic fixed point of $G$. Suppose that there exists a purely atomic s-conformal ending measure $\mu$ for $G$ essentially supported on $G(\zeta)$. By Proposition [5.1. we then have that $\zeta$ does not belong to $L_{H}(G)$, and hence, $\mu$ is essentially supported on $L(G) \backslash L_{H}(G)$. This shows that (i) implies (ii).

For the reverse implication, suppose that there is an $s$-conformal ending measure $\mu$ for $G$ such that $\mu\left(L(G) \backslash L_{H}(G)\right)>0$. By applying Proposition 5.3 to the situation where $A=L(G)$, it follows that there exists a purely atomic $s$-conformal ending measure for $G$ supported on $L(G)$ whose atoms are not parabolic fixed points of $G$. 


\section{EXAMPLES OF CONFORMAL ENDING MEASURES}

In this section we discuss three non-trivial examples of purely atomic, as well as non-atomic conformal ending measures. The first example considers infinitely generated Schottky groups which admit purely atomic conformal ending measures with atoms at Jørgensen points. The second example looks at normal subgroups of finitely generated Schottky groups. We show that for groups of this type there can be an uncountable family of mutually singular non-atomic conformal ending measures. The third example considers a Kleinian group $\Gamma$ which admits a purely atomic $\delta(\Gamma)$-conformal ending measure at a Jørgensen point which is not a parabolic fixed point.

Example 1. Atomic measures at Jørgensen points of infinitely generated Schottky groups.

We consider a particular class of infinitely generated classical Schottky groups (see also [18]). In order to define these groups, let us fix some increasing function $\phi: \mathbb{N} \rightarrow \mathbb{R}^{+}$such that $\phi$ is compatible with the following inductive construction of the set $\mathcal{C}=\left\{C_{n}^{\epsilon}: \epsilon \in\{+,-\}, n \in \mathbb{N}\right\}$ of pairwise disjoint open $N$-dimensional discs $C_{n}^{ \pm}$in $\mathbb{S}$ with radii $r_{n}^{ \pm}$. For ease of exposition, let us assume that $r_{n}:=r_{n}^{+}=r_{n}^{-}<1$, for all $n \in \mathbb{N}$. With $C_{n}^{ \pm, \phi}\left(\supset C_{n}^{ \pm}\right)$denoting the disc concentric to $C_{n}^{ \pm}$of radius $\phi(n) r_{n}$, we now choose the elements in $\mathcal{C}$ by way of induction as follows. Let $C_{1}^{+}$ and $C_{1}^{-}$be chosen such that $C_{1}^{+, \phi} \cap C_{1}^{-, \phi}=\emptyset$. For the inductive step, assume that $C_{1}^{+}, C_{1}^{-}, \ldots, C_{n}^{+}, C_{n}^{-}$have been constructed for some $n \in \mathbb{N}$. Then choose $C_{n+1}^{+}$ and $C_{n+1}^{-}$such that

$$
C_{n+1}^{+, \phi} \cap C_{n+1}^{-, \phi}=\emptyset \text { and } C_{n+1}^{\epsilon, \phi} \cap C_{k}^{\epsilon, \phi}=\emptyset \text { for all } k \in\{1, \ldots, n\}, \epsilon \in\{+,-\} .
$$

With these discs at hand, we then define hyperbolic transformations $g_{n}^{\epsilon} \in \operatorname{Con}(N)$, for each $n \in \mathbb{N}$ and $\epsilon \in\{+,-\}$, such that $g_{n}^{-}=\left(g_{n}^{+}\right)^{-1}$ and

$$
g_{n}^{+}\left(\operatorname{Ext}\left(C_{n}^{+}\right)\right)=\operatorname{Int}\left(C_{n}^{-}\right) \text {and } g_{n}^{-}\left(\operatorname{Ext}\left(C_{n}^{-}\right)\right)=\operatorname{Int}\left(C_{n}^{+}\right) .
$$

Here, Int and Ext denote respectively the interior and exterior in the topology of S. Then let $G^{\phi}$ be the group generated by the set

$$
G_{1}^{\phi}=\left\{g_{n}^{\epsilon}: \epsilon \in\{+,-\}, n \in \mathbb{N}\right\} .
$$

One easily verifies that $\bigcap_{C_{n}^{\epsilon} \in \mathcal{C}} \operatorname{Ext}\left(C_{n}^{\epsilon}\right)$ is a fundamental domain for the action of $G^{\phi}$ on $\mathbb{S}$ (cf. [9], Proposition VIII.A.4), which shows in particular that $G^{\phi}$ is a Kleinian group. Note that the set $L_{J}\left(G^{\phi}\right)$ of Jørgensen limit points contains the accumulation points of $\left\{C_{n}^{\epsilon} \in \mathcal{C}\right\}$. Then let $L_{J}^{\prime}\left(G^{\phi}\right)$ denote the subset of $L_{J}\left(G^{\phi}\right)$ given by

$$
L_{J}^{\prime}\left(G^{\phi}\right)=L_{J}\left(G^{\phi}\right) \cap \bigcap_{\epsilon \in\{+,-\}, n \in \mathbb{N}} \operatorname{Ext}\left(C_{n}^{\epsilon, \phi}\right) .
$$

Clearly, by construction we have that $L_{J}^{\prime}\left(G^{\phi}\right) \neq \emptyset$.

Proposition 6.1. Let $s \in \mathbb{R}^{+}$be given, and let $\phi_{s}: \mathbb{N} \rightarrow[2, \infty)$ be an increasing function such that $\sum_{n \in \mathbb{N}}\left(4 / \phi_{s}(n)\right)^{2 s}<1 / 2$. We then have that the horospherical Poincaré series for $G^{\phi_{s}}$ at $\zeta$ converges at the exponent $s$, for each $\zeta \in L_{J}^{\prime}\left(G^{\phi_{s}}\right)$. Moreover, the essential support of the associated s-conformal ending measure $\mu_{\zeta}$ is equal to $G^{\phi_{s}}(\zeta)$. 
Proof. First note that for each $\zeta \in \operatorname{Ext}\left(C_{n}^{\epsilon, \phi_{s}}\right), g_{n}^{\epsilon} \in G_{1}^{\phi_{s}}$ and $\epsilon \in\{+,-\}$, we have that

$$
\left|\zeta-\left(g_{n}^{\epsilon}\right)^{-1}(0)\right| \geq \phi_{s}(n) r_{n}-r_{n}=\left(\phi_{s}(n)-1\right) r_{n} .
$$

Using this together with $\phi_{s}(n) \geq 2$ and the fact that $1-\left|g_{n}^{\epsilon}(0)\right| \leq 2 r_{n}^{2}$, it follows, for each $\zeta \in \operatorname{Ext}\left(C_{n}^{\epsilon, \phi_{s}}\right)$,

$$
j\left(g_{n}^{\epsilon}, \zeta\right)=\frac{1-\left|g_{n}^{\epsilon}(0)\right|^{2}}{\left|\left(g_{n}^{\epsilon}\right)^{-1}(0)-\zeta\right|^{2}} \leq \frac{2\left(1-\left|g_{n}^{\epsilon}(0)\right|\right)}{\left(\phi_{s}(n)-1\right)^{2} r_{n}^{2}} \leq \frac{4}{\left(\phi_{s}(n)-1\right)^{2}} \leq\left(\frac{4}{\phi_{s}(n)}\right)^{2} .
$$

Then note that every element $\gamma \in G^{\phi_{s}}$ is uniquely represented by a reduced word $g_{n_{1}(\gamma)}^{\epsilon_{1}} \cdots g_{n_{k}(\gamma)}^{\epsilon_{k}}$ of elements in $G_{1}^{\phi_{s}}$. As usual, $k$ will be referred to as the word length of $\gamma$. With $G_{k}^{\phi_{s}}$ denoting the set of elements of $G^{\phi_{s}}$ of word length $k \in \mathbb{N}$, we then obtain for $\zeta \in L_{J}^{\prime}\left(G^{\phi_{s}}\right)$, using the chain rule,

$$
\begin{aligned}
\sum_{\gamma \in G^{\phi_{s} \backslash\{\text { id. }\}}} j(\gamma, \zeta)^{s} & =\sum_{k \in \mathbb{N}} \sum_{\gamma \in G_{k}^{\phi_{s}}} j\left(g_{n_{1}(\gamma)}^{\epsilon_{1}} \cdots g_{n_{k}(\gamma)}^{\epsilon_{k}}, \zeta\right)^{s} \\
& =\sum_{k \in \mathbb{N}} \sum_{\gamma \in G_{k}^{\phi_{s}}} \prod_{i=1}^{k} j\left(g_{n_{i}(\gamma)}^{\epsilon_{i}}, g_{n_{i+1}(\gamma)}^{\epsilon_{i+1}} \cdots g_{n_{k}(\gamma)}^{\epsilon_{k}}(\zeta)\right)^{s} \\
& \leq \sum_{k \in \mathbb{N}} \sum_{\gamma \in G_{k}^{\phi_{s}}} \prod_{i=1}^{k}\left(\frac{4}{\phi_{s}\left(n_{i}(\gamma)\right)}\right)^{2 s} \\
& \leq \sum_{k \in \mathbb{N}}\left[2 \sum_{n \in \mathbb{N}}\left(\frac{4}{\phi_{s}(n)}\right)^{2 s}\right]^{k}<\infty .
\end{aligned}
$$

This shows that the horospherical Poincare series for $G^{\phi_{s}}$ at $\zeta$ converges at the exponent $s$. Applying Theorem 4.3 to the associated $s$-conformal ending measure $\mu_{\zeta}$, finishes the proof of the proposition.

Example 2. Non-atomic conformal ending measures for normal subgroups of Schottky groups.

Here, we consider a class of infinitely generated Kleinian groups which has already been studied in [7. Let $G=G_{1} * G_{2}$ denote the Schottky group obtained as the free product of the two finitely generated non-elementary Schottky groups $G_{1}$ and $G_{2}$. Assume that $\delta(G)>N / 2$, and that $G_{1}$ and $G_{2}$ have Dirichlet fundamental domains $F_{1}$ and $F_{2}$ at $0 \in \mathbb{D}$ such that $\left(\mathbb{D} \backslash F_{1}\right) \cap\left(\mathbb{D} \backslash F_{2}\right)=\emptyset$. Then $G$ is isomorphic to the semidirect product $\Gamma \rtimes G_{2}$, where $\Gamma$ denotes the kernel of the canonical group homomorphism from $G$ onto $G_{2}$. By the splitting lemma for groups, this is equivalent to the existence of a short exact sequence of group homomorphisms

$$
1 \rightarrow \Gamma \rightarrow G \rightarrow G_{2} \rightarrow 1 .
$$

Then note that, since $\Gamma$ is normal in $G$, we have that $L(G)=L(\Gamma)$. Moreover, by a result of Brooks [ $[$, we have that $\delta(\Gamma)<\delta(G)$. Finally, note that by construction we have that $L\left(G_{2}\right) \subset L_{J}(\Gamma)$.

Proposition 6.2. For $G, G_{1}, G_{2}$ and $\Gamma$ given as above, the following hold:

(i) $\Gamma$ is of convergence type. 
(ii) Any $\delta(\Gamma)$-conformal measure for $\Gamma$ is atomless. In particular, for each $\zeta \in L_{J}(\Gamma)$, the $\delta(\Gamma)$-conformal ending measure $\mu_{\zeta}$ for $\Gamma$ derived from some ending sequence towards $\zeta$ has no atoms.

(iii) If $\mu_{\zeta_{1}}$ and $\mu_{\zeta_{2}}$ are two $\delta(\Gamma)$-conformal ending measures for $\Gamma$, for distinct $\zeta_{1}, \zeta_{2} \in L\left(G_{2}\right)$, then $\mu_{\zeta_{1}}$ and $\mu_{\zeta_{2}}$ are mutually singular.

Proof. For the proof of (i), note that Corollary 4.3 in [12] states that if $\Gamma^{\prime}$ is a Kleinian group of divergence type which is normal in some other Kleinian group $G^{\prime}$, then we have that $\delta\left(\Gamma^{\prime}\right)=\delta\left(G^{\prime}\right)$ and that $G^{\prime}$ is of $\delta\left(G^{\prime}\right)$-divergence type. Therefore, assuming that $\Gamma$ is of $\delta(\Gamma)$-divergence type would imply that $\delta(\Gamma)=\delta(G)$, and this is clearly not the case. This finishes the proof of (i).

For the proof of (ii), note that Theorem 6 in 10] states that if $\Gamma^{\prime}$ is a non-trivial normal subgroup of a Kleinian group $G^{\prime}$, then $L_{r}\left(G^{\prime}\right) \subset L_{H}\left(\Gamma^{\prime}\right)$. Hence for our situation here, where $\Gamma$ is normal in $G$ and where $G$ is a Schottky group, it follows that $L(\Gamma)=L(G)=L_{r}(G) \subset L_{H}(\Gamma)$ and hence, we can deduce $L(\Gamma)=L_{H}(\Gamma)$. An application of Proposition 5.1 in Section 5 then gives that any $\delta(\Gamma)$-conformal measure for $\Gamma$ is atomless (note that we have by construction that $\Gamma$ does not have parabolic elements). This completes the proof of (ii).

Finally, in order to prove (iii), let $\zeta_{1}, \zeta_{2} \in L\left(G_{2}\right)$ be given such that $\zeta_{1} \neq \zeta_{2}$. Since $G_{2}$ is a finitely generated Schottky group, it is not difficult to see that there are hyperplanes in $\mathbb{D}$, denoted $H_{1}$ and $H_{2}$, which are at the boundary of some image of the Dirichlet fundamental domain $F_{2}$ of $G_{2}$, such that the following holds. First, $H_{1}$ separates $\mathbb{D}$ into half-spaces one of which has $\zeta_{1}$ at infinity, and the other contains $H_{2}$ and has $\zeta_{2}$ at infinity; secondly, the analogous condition holds for $H_{2}$. By construction of $\Gamma$, it now follows that $H_{1}$ and $H_{2}$, when projected to $\bar{M}_{\Gamma}=(\mathbb{D} \cup \Omega(\Gamma)) / \Gamma$, separate distinct 'ends with boundary' as introduced in [1, Subsection 4.3. Since Theorem 4.4 in [1] holds for such ends with boundary as well, we can conclude that the measures $\mu_{\zeta_{1}}$ and $\mu_{\zeta_{2}}$ have distinct essential supports and thus are mutually singular.

Example 3. Atomic $\delta(\Gamma)$-conformal ending measures at limit points which are not fixed points.

We construct a Kleinian group $\Gamma$ for which there is a purely atomic $\delta(\Gamma)$ conformal ending measure at a Jørgensen point of $\Gamma$ which is not a parabolic fixed point. As in Example 2, let $G=G_{1} * G_{2}$ such that we have an exact sequence of group homomorphisms

$$
1 \rightarrow \Gamma \rightarrow G \rightarrow G_{2} \rightarrow 1 .
$$

This time $G_{2}$ denotes a parabolic Abelian subgroup of $\operatorname{Con}(N)$ with fixed point equal to $\zeta$, and $G_{1}<\operatorname{Con}(N)$ is a non-elementary Kleinian group with $\Omega\left(G_{1}\right) \neq$ $\emptyset$. As in the previous example, let us assume that $G_{1}$ and $G_{2}$ have Dirichlet fundamental domains $F_{1}$ and $F_{2}$ at $0 \in \mathbb{D}$ such that $\left(\mathbb{D} \backslash F_{1}\right) \cap\left(\mathbb{D} \backslash F_{2}\right)=\emptyset$. Moreover, we assume that $\delta(G)>N / 2$, and that $G$ is of convergence type. Clearly, the latter condition is always satisfied, for instance, if we choose $G_{1}$ such that $\delta\left(G_{1}\right)=N$. Then note that, since $G / \Gamma$ is isomorphic to the amenable group $G_{2}$, an application of a result of [6] gives us that $\delta(\Gamma)=\delta(G)$. Also, note that $\zeta$ is a bounded parabolic fixed point of $G$, whereas $\zeta$ is a Jørgensen limit point of $\Gamma$ which is not fixed by any non-trivial element of $\Gamma$. 
Proposition 6.3. For $G, G_{1}, G_{2}, \Gamma$ and $\zeta$ given as above, we have that the $\delta(\Gamma)$ conformal ending measure $\mu_{\zeta}$ for $\Gamma$ is essentially supported on $\Gamma(\zeta)$. In particular, we hence have that $\mu_{\zeta}$ is purely atomic.

Proof. By Lemma 4.4, we have that the reduced horospherical Poincaré series

$$
\mathcal{P}_{\text {red }}(\zeta, s)=\sum_{g \in G / \operatorname{Stab}_{G}(\zeta)} j(g, \zeta)^{s}
$$

for $G$ converges for $s$ equal to the exponent $\delta(G)$ since we assume that $G$ is of convergence type. Recall that $\delta(G)=\delta(\Gamma)$, and that $\operatorname{Stab}_{G}(\zeta)=G_{2}$. We now show that the horospherical Poincaré series $\sum_{\gamma \in \Gamma} j(\gamma, \zeta)^{\delta(\Gamma)}$ for $\Gamma$ at the exponent $\delta(\Gamma)$ converges. Indeed, since $G=\Gamma \rtimes G_{2}$, we can take $\Gamma$ as a system of representatives of $G / \operatorname{Stab}_{G}(\zeta)$. With this choice, we then have

$$
\sum_{g \in G / \mathrm{Stab}_{G}(\zeta)} j(g, \zeta)^{\delta(G)}=\sum_{\gamma \in \Gamma} j(\gamma, \zeta)^{\delta(\Gamma)} .
$$

Therefore, the horospherical Poincare series for $\Gamma$ at the exponent $\delta(\Gamma)$ converges, and hence we can apply Theorem 4.3, which then gives the existence of a purely atomic $\delta(\Gamma)$-conformal ending measure for $\Gamma$ which is essentially supported on $\Gamma(\zeta)$.

\section{REFERENCES}

1. J. W. Anderson, K. Falk and P. Tukia, Conformal measures associated to ends of hyperbolic n-manifolds, Quart. J. Math. 58 (2007), 1-15. MR2305045

2. A. F. Beardon, The geometry of discrete groups, Springer Verlag, New York, 1983. MR698777 (85d:22026)

3. A. F. Beardon and B. Maskit, Limit points of Kleinian groups and finite sided fundamental polyhedra, Acta Math. 132 (1974), 1-12. MR0333164 (48:11489)

4. C. Bishop, On a theorem of Beardon and Maskit, Ann. Acad. Sci. Fenn. Math. 21 (1996), 383-388. MR1404092 (97f:30064)

5. C. J. Bishop and P. W. Jones, Hausdorff dimension and Kleinian groups, Acta Math. 56 (1997), 1-39. MR.1484767 (98k:22043)

6. R. Brooks, The bottom of the spectrum of a Riemannian cover, J. Reine Angew. Math. 357 (1985), 101-114. MR783536 (86h:58138)

7. K. Falk and B. O. Stratmann, Remarks on Hausdorff dimensions for transient limit sets of Kleinian groups, Tohoku Math. J. (2), 56 (2004), 571-582. MR2097162 (2005g:30053)

8. K. Falk and P. Tukia, A note on Patterson measures, Kodai Math. J. 29 (2006), 227-236. MR 2247432 (2007j:30036)

9. B. Maskit, Kleinian groups, Springer Verlag, Berlin, 1989. MR959135 (90a:30132)

10. K. Matsuzaki, Conservative action of Kleinian groups with respect to the Patterson-Sullivan measure, Comp. Meth. Funct. Th. 2 (2002), 469-479. MR2038133(2005h:30083)

11. K. Matsuzaki and M. Taniguchi, Hyperbolic manifolds and Kleinian groups, Oxford Math. Monographs, 1998. MR.1638795 (99g:30055)

12. K. Matsuzaki and Y. Yabuki, The Patterson-Sullivan measure and proper conjugation for Kleinian groups of divergence type, Erg. Th. Dyn. Syst. 29 (2009), 657-665. MR2486788

13. S. Morosawa, Invariant subsets of the limit set for a Fuchsian group, Tohoku Math. J. (2), 42 (1990), 429-437. MR1066671 (92a:30042)

14. P. J. Nicholls, The ergodic theory of discrete groups, LMS Lecture Notes Series Vol. 143, Cambridge Univ. Press, 1989. MR1041575 (91i:58104)

15. J. R. Parker, B. O. Stratmann, Kleinian groups with singly cusped parabolic fixed points, Kodai J. Math. 24 (2001), 169-206. MR1839255(2002e:20101)

16. S. J. Patterson, The limit set of a Fuchsian group, Acta Math. 136 (1976), 241-273. MR 0450547 (56:8841) 
17. B. O. Stratmann, The exponent of convergence of Kleinian groups; on a theorem of Bishop and Jones, Progress in Probability, Vol. 57, Birkhäuser Verlag, 93-107, 2004. MR.2087134 (2005h:20114)

18. B. O. Stratmann, M. Urbański, Pseudo-Markov systems and infinitely generated Schottky groups, Amer. J. Math. 129 (2007), 1019-1063. MR2343382 (2008j:37050)

19. D. Sullivan, The density at infinity of a discrete group of hyperbolic motions, IHES Publ. Math. 50 (1979), 171-202. MR556586 (81b:58031)

20. D. Sullivan, On the ergodic theory at infinity of an arbitrary discrete group of hyperbolic motions, Riemann surfaces and related topics, Ann. Math. Studies Vol. 97, Princeton University Press, 465-496, 1981. MR624833 (83f:58052)

21. D. Sullivan, Related aspects of positivity in Riemannian Geometry, J. Differential Geom. 25 (1987), 327-351. MR882827 (88d:58132)

22. P. Tukia, Conservative action and the horospheric limit set, Ann. Acad. Sci. Fenn. 22 (1997), 387-394. MR1469798 (98i:30057)

Fachbereich 3 - Mathematik und Informatik, Universität Bremen, Bibliothekstr. 1, D-28359 Bremen, Germany

E-mail address: khf@math.uni-bremen.de

Department of Mathematics, School of Education, Waseda University, Shinjuku, TOKYO 169-8050, JAPAN

E-mail address: matsuzak@waseda.jp

Fachbereich 3 - Mathematik und Informatik, Universität Bremen, Bibliothekstr. 1, D-28359 Bremen, Germany

E-mail address: bos@math.uni-bremen.de 